\title{
Survival of the Fittest? Examining Lapsing Behaviour in the Context of Elderly People and the Use of Physical Activity Tracker Applications
}

\author{
Markus Makkonen \\ ${ }^{1}$ Institute for Advanced \\ Management Systems Research \\ ${ }^{2}$ University of Jyvaskyla \\ markus.v.makkonen@jyu.fi
}

\author{
Tuomas Kari \\ ${ }^{1}$ Institute for Advanced \\ Management Systems Research \\ ${ }^{2}$ University of Jyvaskyla \\ tuomas.t.kari@jyu.fi
}

\author{
Lauri Frank \\ University of Jyvaskyla \\ lauri.frank@j.jyu.fi
}

\begin{abstract}
Physical activity (PA) tracker applications have been proposed as one potential solution to the increasingly prevalent physical inactivity problem among elderly people, but their long-term potential is limited by the frequent lapses in their use. In this study, our objective is to promote the understanding of the lapsing behaviour of PA tracker applications among elderly people. More specifically, we are interested in how gender, age, and household type as well as initial PA level and technology readiness (TR) affect the risk of lapsing. As the data for the study, we use actual PA tracker application usage data as well as survey data, which were both collected in our ongoing research program and are analysed by using survival analysis. We find lapsing behaviour to be affected mainly by initial PA level as well as to some degree by TR and gender but not by age and household type.
\end{abstract}

\section{Introduction}

In several studies, increased physical activity (PA) and decreased sedentary behaviour have been found to provide notable health benefits also in older age [1]. Nevertheless, many elderly people fail to meet the PA guidelines recommended by the public health agencies such as the World Health Organisation [2]-[3]. During the past two years, this physical inactivity problem appears to have been further aggravated by the ongoing coronavirus disease 2019 (COVID-19) pandemic caused by the severe acute respiratory syndrome coronavirus 2 (SARS-CoV-2) [4]-[5], which has limited the possibilities for PA especially among elderly people. Thus, new ways to promote the PA levels among elderly people are urgently needed. One potential way to do this are different kinds of PA tracker applications, which enable users to keep track of their PA in everyday life and potentially change their behaviour based on the tracked data. These kinds of applications have been found very promising in terms of promoting the PA levels not only in the general population (e.g., [6]-[8]) but also in the elderly population (e.g., [9][11]), although more high-quality studies especially on their long-term effects are still called for. In addition to elderly people in general, their potential has been highlighted particularly in the more specific segment of young elderly [12-15], which consists of people aged approximately $60-75$ years.

However, a well-known issue with the use of PA tracker applications, as well as many other personal informatics and self-tracking technologies, is that instead of using them adherently, people either abandon them quickly or have frequent lapses and resumptions in their use [16]-[20], which limits especially their long-term potential [21]-[22]. To make things worse, we also have a very limited understanding of the actual reasons behind these phenomena. For example, the factors affecting lapsing and abandonment behaviour have been studied only in a few prior studies (e.g., [16]-[17]) and mainly qualitatively rather than quantitatively [21], with none of them focusing specifically on the context of elderly people. On adherence behaviour, more studies with more varied methodological approaches are available (cf. [20]), but as above, none of them have focused specifically on the context of elderly people and most have been relatively short-term rather than long-term studies. Thus, there seems to be a clear call for studies that focus on lapsing, abandonment, and adherence behaviour especially among elderly people and in long-term settings.

In the present study, our objective is to address the aforementioned call by examining lapsing behaviour in the context of elderly people, by whom we refer to people aged 60 years or over, and the use of PA tracker applications. More specifically, we are interested in how gender, age, and household type as well as initial $P A$ level and technology readiness affect the risk of lapsing. The reasons for focusing on these particular five factors will be discussed in more detail in the next 
section of the paper. As the data for the study, we use actual PA tracker application usage data from our ongoing research program, in which the participants are provided with a PA tracker application and asked to use it to keep track of their PA in everyday life. In the case of some of the participants, this data spans over two years, thus enabling the examination of not only short-term but also long-term lapsing behaviour. In addition, we also collect survey data from the participants. The collected data is analysed by using survival analysis, which to our knowledge, has not previously been used to examine lapsing behaviour in the context of personal informatics and self-tracking.

After this introductory section, we will present the theoretical foundation of the study in Section 2. This is followed by a description of the research methodology and reporting of the research results in Sections 3 and 4 . The results will be discussed in more detail in Section 5. Finally, we will conclude the paper with a brief discussion about the limitations of the study and some potential paths of future research in Section 6 .

\section{Theoretical foundation}

In this section, we will first discuss in more detail the concepts of lapsing, abandonment, adherence, and technology readiness as well as propose our research hypotheses, which will be later tested in accordance with the traditional hypothetico-deductive model.

\subsection{Lapsing, abandonment and adherence}

In the context of personal informatics and selftracking, the concept of lapsing has been highlighted especially in the lived informatics model of personal informatics by Epstein et al. [16], which is an extension of the model of personal informatics by Li et al. [23]. In the model by Epstein et al. [16], the self-tracking process is hypothesised to consist of five subsequent stages: (1) deciding to track, (2) selecting the tools, (3) tracking and acting, (4) lapsing, and (5) resuming. Of these, the first three stages correspond to the five original stages of the model by Li et al. [23]: (1) preparation, (2) collection, (3) integration, (4) reflection, and (5) action. During these stages, a person first makes the decision to track some aspect of his or her life, then selects the appropriate tools for this, and finally conducts the actual tracking in a continuous cycle of collecting, integrating, and reflecting the data. In contrast, the two latter stages extend the model by Li et al. [23] by acknowledging that in the context of lived informatics, which refers to the real-life use of personal informatics in everyday life [24], the selftracking process is typically characterised by frequent lapses and resumptions, in which people temporarily stop the tracking activities and then start them again. Of course, in some cases, the temporary lapses may become permanent, resulting in the abandonment of the tracking activities altogether.

However, although lapsing and abandonment are seen as an integral part of lived informatics, the reasons for them have been examined only in a few prior studies and mainly qualitatively rather than quantitatively [21]. For example, Epstein et al. [16] have found that the main reasons for lapsing are accidentally forgetting to track, upkeeping the tracking tools, intentionally skipping tracking, or temporarily suspending tracking. In turn, Epstein et al. [17] have found that the main reasons for abandonment are the cost of collecting and integrating the data, the cost of having or sharing the data, discomfort with the revealed information, data quality concerns, having learned enough, and changes in life circumstances.

A closely connected concept to lapsing and abandonment is adherence, which basically refers to how long one is able to maintain the tracking activities without lapsing or abandonment [19]. In contrast to lapsing and abandonment, adherence has been examined in more numerous prior studies both qualitatively and quantitatively, of which many have also focused specifically on the context of PA tracker applications (cf. [20]). Although none of these prior studies have focused specifically on the context of elderly people and most of them have been relatively short-term rather than long-term studies, their findings can be used as a basis for the research hypotheses of our study. Based on a literature review of these prior studies, Yang et al. [20] have classified the factors that affect the adherence to PA tracker applications into three main categories: personal factors, technology features, and contextual factors. Of these, in this study, we will focus on personal factors, more specifically three demographic factors and two psychological factors.

The three demographic factors are gender, age, and household type, in the case of which the adherence to PA tracker applications has been found to be higher for men [25] and older people [25]-[26] but lower for those living in households of only one adult [26]. Of these, the higher adherence of men can be explained by their more positive attitude towards technology [25], whereas the higher adherence of older people can be explained by their greater engagement with PA interventions [25]. In turn, the lower adherence of those living in households of only one adult is most likely explained by their lack of social support and social interaction, which have been found important in terms of improving the adherence to PA interventions [26]. Thus, based on these findings, we propose the following three hypotheses concerning the effects of gender, age, and household type on lapsing behaviour: 
H1: Women have a higher risk of lapsing in comparison to men.

H2: Older people have a lower risk of lapsing in comparison to younger people.

H3: People living alone have a higher risk of lapsing in comparison to people living with others.

In turn, the first psychological factor is the attitude towards health and fitness, in the case of which the adherence to PA tracker applications has been found to be higher for people with a more positive attitude and lower for people with a less positive attitude [27]. However, in this study, instead of measuring the attitude towards health and fitness directly, we measure it indirectly via the proxy of initial PA level, with which we refer to the PA level at the beginning of the tracking activities. Here, the underlying assumption is that having a higher initial PA level is associated with a more positive attitude towards health and fitness, whereas having a lower initial PA level is associated with a less positive attitude towards health and fitness. Thus, we propose the following hypothesis concerning the effect of initial PA level on lapsing behaviour:

H4: People who have a higher initial PA level have a lower risk of lapsing in comparison to people who have a lower initial PA level.

\subsection{Technology readiness}

The second psychological factor is technology readiness (TR), which refers to people's propensity to embrace and use new technologies for accomplishing goals in home life and at work [28]. It is typically seen as comprising of four dimensions [28]: (1) optimism (i.e., a positive view of technology and a belief that it offers people increased control, flexibility, and efficiency in their lives), (2) innovativeness (i.e., a tendency to be a technology pioneer and thought leader), (3) discomfort (i.e., a perceived lack of control over technology and a feeling of being overwhelmed by it), and (4) insecurity (i.e., distrust of technology and scepticism about its ability to work properly). TR has been found to affect the adoption and use of especially self-service technologies (e.g., [29]-[31]) but also fitness, dietary, and health applications as well as sports wearable technologies (e.g., [32]-[34]). TR has also been found to affect technology adoption and use among not only younger people but also elderly people (e.g., [35]-[36]) as well as post-adoption behaviours like use continuance (e.g., [37]). For example, Chen et al. [37] have found especially the positive optimism and innovativeness dimensions of TR to affect use continuance both directly as well as indirectly via satisfaction, perceived usefulness, and perceived ease of use, whereas the negative discomfort and insecurity dimensions of TR were found to have no effects.

This all suggests that TR may very well act as an important psychological factor affecting also the lapsing behaviour of PA tracker applications among elderly people. Thus, based on the aforementioned findings by Chen et al. [37] on use continuance, which can be considered a closely connected concept to lapsing, we propose the following four hypotheses concerning the effects of TR on lapsing behaviour:

H5: People with a higher level of optimism have a lower risk of lapsing in comparison to people with a lower level of optimism.

H6: People with a higher level of innovativeness have a lower risk of lapsing in comparison to people with a lower level of innovativeness.

H7: The level of discomfort has no effect on the risk of lapsing.

H8: The level of insecurity has no effect on the risk of lapsing.

\section{Methodology}

This study was conducted as part of our ongoing DigitalWells research program, which uses digital wellness technologies to study and promote the PA of young elderly in Finland. The multiyear and nationwide program is conducted in close co-operation with Finnish pensioners' associations, which are responsible for recruiting volunteer participants to the program amongst their members. Participation in the program is free, but each participant must own a smartphone. As part of the program, the participants are provided for free with both a PA tracker application as well as the training and support for setting up and using it, and they are asked to use it to keep track of their PA in everyday life. The application is developed by the program itself on top of the Wellmo [38] platform, and it is available for both Google's Android and Apple's iOS operating systems. The logging of PA in the application is conducted mainly manually by entering the type, intensity, time, and duration of each PA. Currently, the application supports 35 different types of PA (+ "other activity"), which include both sports (e.g., jogging and gym training) and non-sports (e.g., household work and yard work) activities. In addition, the application has the ability to import the loggings automatically from other services, such as Google Fit, Apple Health, and Polar Flow. Based on the loggings, 
the application shows the participants different types of reports about their PA on a weekly, monthly, and yearly basis. More information about the application is available from the authors upon request.

In this study, we use the loggings that were made by the participants by 31 May 2021. In other words, this date is the fixed end date of the observation period for all the participants. In turn, the start date of the observation period for each participant is the date on which the participant made his or her first logging. This is not a fixed day but varies between the participants because they typically entered the program in groups of about 20-40 people at a time. Due to this variation, also the duration of the observation period is not fixed but varies between the participants. However, we excluded the participants who had entered the program and made their first logging after 30 April 2021, meaning that for all the participants, the duration of the observation period was at least one month.

In addition to using the application, the participants were also asked to complete an entry survey when they entered the program and multiple follow-up surveys, of which the first one was conducted about four months after the entry survey and the following ones about six months after the previous follow-up survey. Originally, these were pen-and-paper surveys that were filled in the face-to-face group meetings, but after the outbreak of the COVID-19 pandemic, all the surveys were conducted as online surveys. The complete questionnaires of the surveys are available from the authors upon request. Gender, age, household type, and initial PA level were each measured in the entry survey by using a single item. Of these, gender $(0=$ man, $1=$ woman $)$ and household type $(0=$ living alone, $1=$ living with others) were measured by using closed-ended questions with two options, whereas age at the beginning of the observation period (in years) was approximated based on an open-ended question measuring the year of birth. In turn, the self-assessed initial PA level was measured by using a closed-ended question with six options ( 1 = very high, $2=$ high, $3=$ moderate, $4=$ low, $5=$ very low, $6=$ totally passive), but the responses to this question were later recoded into two dummy variables indicating whether $(0=$ no, $1=$ yes) the initial PA level was high (options 1-2) or low (options 4-6). Finally, TR was measured either in the entry survey or in one of the first two follow-up surveys by using the 16 items of the technology readiness index (TRI) 2.0 scale [39] and a five-point Likert scale. Each of the TR dimensions was measured by using four items, the scores of which were averaged to calculate the score of each TR dimension. Because Finland has two official languages, the participants had the option to respond to the surveys in either Finnish or Swedish. The participants who did not respond to the questions concerning their gender, age, household type, or initial PA level or to at least one item measuring each of the four TR dimensions were excluded from the study. In addition, we excluded five participants who were aged under 60 years due to our focus on the elderly segment.

The collected data was analysed by using survival analysis, which has traditionally been used especially in medical research. Although not previously used to examine lapsing behaviour, survival analysis can be considered an appropriate statistical technique also for this purpose because it is interested in the survival times of people until the occurrence of a particular event, such as a disease or death. In this case, that particular event is obviously lapsing, meaning that we are interested in how long a particular user of a PA tracker application is able to "survive" before a lapse. More specifically, we use the Prentice, Williams, and Peterson [40] model with gap times (PWP-GT), which is an extension of the traditional Cox [41] proportional hazards regression model (Cox $\mathrm{PH}$ ). Both these models are used to analyse the effects of one or more covariates on event probabilities (i.e., hazards) based on the proportional hazards assumption of the effect of each covariate remaining constant over time. However, whereas the Cox PH model is limited to the case in which the events occur only once, the PWP-GT model is applicable also to the case of recurrent events, such as the case of lapsing, in which a user may resume from a lapse and then relapse. The PWP-GT model also allows varying baseline hazards for the events in terms of their occurrence number. This means that, for example, the baseline hazard of the event occurring for the first time may be lower or higher in comparison to the baseline hazard of the event recurring. This is likely to be useful in the case of lapsing behaviour because the baseline risk of relapsing may be higher than the baseline risk of lapsing for the first time, and it may even become higher with each relapse.

Before conducting the actual analyses, the loggings that were made with the PA tracker application were transformed into survival intervals, which refer to continuous time periods of making loggings without lapsing. The start date of each survival period is the date of the first logging by a participant or the date of his or her first logging after resuming from a lapse, whereas the end date of each survival period is the date of the first day of a lapse. Alternatively, if a participant either had not lapsed for the first time or had not relapsed after resuming from a lapse by the end date of the observation period, the survival interval ended with right censoring at the next day, meaning that we only know that the lapse or relapse had not occurred by 31 May 2021. In other words, each survival interval can end in either lapsing, in which case we have full 
information of its start and end dates, or in censoring, in which case we only have partial information in terms of knowing the start date but not the end date.

We initially operationalised lapsing as a time period of at least seven days with no loggings. This operationalisation was based on the fact that many aspects of human behaviour, including PA, typically occur in a weekly rhythm [21]. Thus, if a participant had not logged any PA for a week, it was very likely that this gap was caused by an actual lapse in using the application rather than by the fact that the participant had not done any PA and had nothing to log. However, of course, especially in our target population of elderly people, the latter case is also possible due to hospitalisations or similar issues. Thus, as a reference, we also replicated our analyses with the 14-day and 28-day operationalisations of lapsing in addition to the aforementioned 7-day operationalisation.

The actual analyses were conducted by using the survival 3.2.11 package of R [42]. When conducting them, we also added an extra control variable as a covariate concerning the logging method in terms of whether a participant had made loggings only by entering them manually or whether at least some of them had been imported automatically from other services $(0=$ only manually, $1=$ also automatically). Because the logging method affects the logging effort, it is also likely to have an effect on the risk of lapsing. When interpreting the results, we use $p<0.05$ as the threshold of statistical significance, but we also note the results that are statistically almost significant in terms of meeting the threshold of $\mathrm{p}<0.1$.

\section{Results}

In total, our sample consists of 608 participants. The descriptive statistics of the sample in terms of gender, age, household type, initial PA level, logging method, as well as the time when a participant had made his or her first logging are reported in Table 1. Due to our research program, most of the participants were young elderly aged approximately $60-75$ years, but there were also some participants who were older than that. All in all, the age of the participants ranged from 60 to 85 years with a mean of 69.6 years and a standard deviation of 4.2 years. In turn, the means and standard deviations (SD) of the scores of the four TR dimensions are reported in Table 2, together with the Cronbach's alphas (CA), which measure the reliability of the scores. As can be seen, on average, the participants had relatively high levels of optimism and insecurity but lower levels of innovativeness and discomfort. With a CA of greater than or equal to 0.7 , the reliability of the scores in the case of all the four TR dimensions can be considered acceptable [43].
Table 1. Sample statistics $(\mathrm{N}=608)$

\begin{tabular}{|c|c|c|}
\hline & $\mathbf{N}$ & $\mathbf{\%}$ \\
\hline Gender & & \\
\hline Man & 219 & 36.0 \\
\hline Woman & 389 & 64.0 \\
\hline Age & & \\
\hline 60-69 years & 301 & 49.5 \\
\hline $70-79$ years & 297 & 48.8 \\
\hline 80 years or over & 10 & 1.6 \\
\hline Household type & & \\
\hline Living alone & 146 & 24.0 \\
\hline Living with others & 462 & 76.0 \\
\hline Initial PA level & & \\
\hline Low & 66 & 10.9 \\
\hline Moderate & 432 & 71.1 \\
\hline High & 110 & 18.1 \\
\hline Logging method & & \\
\hline Only manually & 477 & 78.5 \\
\hline Also automatically & 131 & 21.5 \\
\hline First logging & & \\
\hline May 2019-October 2019 & 112 & 18.4 \\
\hline November 2019-April 2020 & 190 & 31.3 \\
\hline May 2020-October 2020 & 120 & 19.7 \\
\hline November 2020-April 2021 & 186 & 30.6 \\
\hline
\end{tabular}

Table 2. TR statistics

\begin{tabular}{|l|c|c|c|}
\hline & Mean & SD & CA \\
\hline Optimism & 3.423 & 0.852 & 0.775 \\
\hline Innovativeness & 2.886 & 0.970 & 0.774 \\
\hline Discomfort & 2.870 & 0.924 & 0.719 \\
\hline Insecurity & 3.418 & 0.910 & 0.713 \\
\hline
\end{tabular}

In total, the 608 participants had made 213,847 loggings during the observation period, with a mean of 352 and a median of 241 loggings per participant. The first logging was made on 16 May 2019, whereas the last loggings were made on 31 May 2021, which was the end date of the observation period. Some descriptive statistics of the observed survival intervals in the case of the three different operationalisations of lapsing are reported in Table 3. For example, in the case of the 7-day operationalisation, a total of 1,656 survival intervals were observed, of which 1,260 ended with lapsing and 396 ended with censoring due to no lapse occurring during the observation period. The minimum number of observed lapses per participant was zero, meaning that some participants had not lapsed at all during the observation period, whereas the maximum number of observed lapses per participant was 28. The minimum number of days before observing a lapse was one, meaning that there were cases in which 
a participant relapsed immediately after resuming from a lapse, whereas the maximum number of days before observing a lapse was 740 . This latter value was censored, meaning that the longest survival interval did not end with lapsing during the observation period.

Table 3. Survival statistics $\left({ }^{*}=\right.$ censored)

\begin{tabular}{|c|c|c|c|}
\hline & 7-day & 14-day & 28-day \\
\hline Survival intervals & 1,656 & 931 & 720 \\
\hline Ends with lapsing & 1,260 & 507 & 272 \\
\hline Ends with censoring & 396 & 424 & 448 \\
\hline Lapses per participant & & & \\
\hline Minimum & 0 & 0 & 0 \\
\hline Maximum & 28 & 13 & 4 \\
\hline Days before a lapse & & & \\
\hline Minimum & 1 & 1 & 1 \\
\hline Maximum & $740^{*}$ & $742^{*}$ & $742^{*}$ \\
\hline
\end{tabular}

Before conducting the actual analyses, we limited the observed occurrence numbers of the lapses to 13, 6 , and 4 in the case of the 7-day, 14-day, and 28-day operationalisations, respectively, meaning that all higher occurrence numbers in the survival intervals were replaced with the aforementioned values. This was because less than one per cent of the participants had survival intervals with higher occurrence numbers, which was considered too small a percentage for calculating the varying baseline hazards of the PWPGT model. We also checked the proportional hazards assumption of the Cox PH and PWP-GT models by using the test by Grambsch and Therneau [44], which supported it globally in the case of the 7-day $\left(\chi^{2}(10)=\right.$ $12.847, \mathrm{p}=0.232), 14$-day $\left(\chi^{2}(10)=9.848, \mathrm{p}=0.454\right)$, and 28-day $\left(\chi^{2}(10)=5.942, \mathrm{p}=0.820\right)$ operationalisations alike. The results of the actual analyses in terms of the hazard ratio of each covariate and its statistical significance in the case of the three different operationalisations of lapsing are reported in Table 4.

Table 4. Results of survival analysis $(* * *=\mathbf{p}$ $<0.001, * *=\mathbf{p}<0.01, *=\mathbf{p}<0.05,(*)=\mathbf{p}<0.1)$

\begin{tabular}{|l|c|c|c|}
\hline & 7-day & 14-day & 28-day \\
\hline Gender & $0.778^{* * *}$ & $0.835^{(*)}$ & 0.823 \\
\hline Age & 0.988 & 0.997 & 0.986 \\
\hline Household type & 1.040 & 1.198 & 1.157 \\
\hline Low initial PA level & $1.480^{* * *}$ & $1.658^{* * *}$ & $1.784^{* *}$ \\
\hline High initial PA level & $0.741^{*}$ & $0.715^{(*)}$ & 0.787 \\
\hline Optimism & 0.953 & $0.874^{(*)}$ & $0.857^{*}$ \\
\hline Innovativeness & 1.031 & 1.044 & 1.066 \\
\hline Discomfort & 1.006 & 0.951 & 0.954 \\
\hline Insecurity & 0.970 & 0.937 & 0.940 \\
\hline Logging method & $0.835^{*}$ & $0.701^{* *}$ & $0.544^{* * *}$ \\
\hline
\end{tabular}

In the case of categorical covariates, a hazard ratio of less than one means a lower risk, whereas a hazard ratio of greater than one means a higher risk of lapsing in the category coded as one in comparison to the category coded as zero. In the case of scalar covariates, a hazard ratio of less than one means a lower risk, whereas a hazard ratio of greater than one means a higher risk of lapsing with higher values of the covariate. Thus, for example, in the case of the 7-day operationalisation, being a woman, having a high initial PA level, and having automatically imported loggings decreased the risk of lapsing, whereas having a low initial PA level increased the risk of lapsing. For example, in the case of initial PA level, those with a low initial PA level had about 48\% higher risk of lapsing, whereas those with a high initial PA level had about $26 \%$ lower risk of lapsing. In contrast, age, household type, and all the four TR dimensions had no statistically significant effect on the risk of lapsing. In turn, in the case of the 14-day operationalisation, the effects of low initial PA level and logging method remained statistically significant and seemed to become even stronger, whereas the effects of gender and high initial PA level became statistically almost significant. In addition, optimism now had a statistically almost significant effect, with being more optimistic decreasing the risk of lapsing. Finally, in the case of the 28-day operationalisation, the effects of low initial PA level and logging method once again remained statistically significant and seemed to become even stronger, whereas the effects of gender and high initial PA level became statistically not significant. In addition, the effect of optimism remained statistically almost significant.

The goodness of fit of the three estimated models with the data was examined by using the likelihoodratio tests (LRT) and Wald [45] tests, the results of which are reported in Table 5. As can be seen, both the tests suggested a good fit with the data in the case of all the three different operationalisations of lapsing.

Table 5. Results of goodness of fit tests

\begin{tabular}{|c|c|c|c|}
\hline & 7-day & 14-day & 28-day \\
\hline LRT & & & \\
\hline$\chi^{2}$ & 56.162 & 41.396 & 34.353 \\
\hline $\mathrm{df}$ & 10 & 10 & 10 \\
\hline $\mathrm{p}$ & $<0.001$ & $<0.001$ & $<0.001$ \\
\hline Wald & & & \\
\hline$\chi^{2}$ & 44.85 & 44.21 & 36.62 \\
\hline $\mathrm{df}$ & 10 & 10 & 10 \\
\hline $\mathrm{p}$ & $<0.001$ & $<0.001$ & $<0.001$ \\
\hline
\end{tabular}

Finally, Table 6 summarises the results in terms of whether they supported the eight research hypotheses proposed in Section 2. 
Table 6. Summary of the results

\begin{tabular}{|l|c|c|c|}
\hline & 7-day & 14-day & 28-day \\
\hline H1 & No support & No support & No support \\
\hline H2 & No support & No support & No support \\
\hline H3 & No support & No support & No support \\
\hline H4 & Support & Weak support & Partly support \\
\hline H5 & No support & Weak support & Weak support \\
\hline H6 & No support & No support & No support \\
\hline H7 & Support & Support & Support \\
\hline H8 & Support & Support & Support \\
\hline
\end{tabular}

\section{Discussion and conclusions}

In this study, we examined lapsing behaviour in the context of elderly people and the use of PA tracker applications. More specifically, we were interested in how gender, age, and household type as well as initial PA level and TR affect the risk of lapsing. First, we proposed eight hypotheses based on the findings of prior studies on the adherence to PA tracker applications and the effects of TR on use continuance. These hypotheses were then tested by using survival analysis to analyse the actual usage data of 608 Finnish elderly users of a PA tracker application together with survey data. In the analyses, we used three different operationalisations of lapsing: (1) a 7-day, (2) a 14-day, and (3) a 28-day period during which the user has not made any loggings by using the application.

All in all, we observed high variance in the lapsing behaviour of the users. For example, in the case of the 7-day operationalisation, several users had used the application for more than two years without lapsing at all, whereas one user had lapsed as many as 28 times during the observation period. In terms of the hypotheses concerning the demographic factors, we found no support for $\mathrm{H} 1$, which proposed that women have a higher risk of lapsing compared to men. On the contrary, we found women to have a lower risk of lapsing in comparison to men in the case of the 7-day operationalisation, and this effect was found statistically almost significant also in the case of the 14-day operationalisation. Thus, our findings seem to conflict with those by Guertler et al. [25], which may be explained by the fact that although men have been found to have a more positive attitude towards technology, women have been found to have greater engagement with PA interventions [25], and the more positive attitude of men towards technology may also concern mainly younger rather than older men. Similarly, we found no support for $\mathrm{H} 2$, which proposed that older people have a lower risk of lapsing in comparison to younger people. Thus, our findings seem to conflict with those by both Guertler et al. [25] and Hermsen et al. [26], which may be at least partly explained by the much more limited age variation in our study because we focused only on the context of elderly people. Finally, we also found no support for $\mathrm{H} 3$, which proposed that people who live alone have a higher risk of lapsing in comparison to people who live with others. Thus, our findings seem to conflict with those by Hermsen et al. [26], although their findings concerned particularly younger people who do not live with other adults but who have children that still live with them. Due to their age, most of the elderly people in our study obviously did not have children who still live with them.

In turn, in terms of the hypotheses concerning the psychological factors, we found at least partial support for $\mathrm{H} 4$, which proposed that people who have a higher initial PA level have a lower risk of lapsing in comparison to people who have a lower initial PA level. Here, we assumed the initial PA level to be associated with the attitude towards health and fitness. In the case of all the three operationalisations, we found a lower initial PA level to considerably increase the risk of lapsing, by about $64 \%$ on average, whereas the effect of a higher initial PA level decreasing the risk of lapsing was found statistically significant in the case of the 7day operationalisation and statistically almost significant in the case of the 14-day operationalisation, but statistically not significant in the case of the 28-day operationalisation. The decrease in the risk of lapsing was also found more modest, about $25 \%$ on average. Thus, especially having a lower initial PA level, and thus assumably a less positive attitude towards health and fitness, can be seen as a considerable risk factor in terms of increasing the risk of lapsing, which supports the findings by Canhoto and Arp [27]. Of course, one could also argue that the observed gaps in the loggings of those with a low initial PA level in comparison to those with a high initial PA level are not due to actual lapsing but simply due to their lack of PA to log. However, as we have already discussed above, we do not consider this argument very plausible because in order to have no PA to log for a period of seven days, not to mention the periods of 14 or 28 days, one would have to be totally passive, and only one person in our sample assessed his or her initial PA level to be this low.

Finally, in terms of the remaining hypotheses concerning the psychological factors, more specifically TR, we found weak support for H5, which proposed that people with a higher level of optimism have a lower risk of lapsing in comparison to people with a lower level of optimism. This effect was found statistically not significant in the case of the 7-day operationalisation but statistically almost significant in the case of the 14-day and 28-day operationalisations. In turn, we found no support for H6, which proposed that people with a higher level of innovativeness have a 
lower risk of lapsing in comparison to people with a lower level of innovativeness. This effect was found statistically not significant in the case of all the three operationalisations. Finally, we found support for hypotheses $\mathrm{H} 7$ and $\mathrm{H} 8$, which proposed that the levels of discomfort and insecurity have no effects on the risk of lapsing. These effects were found statistically not significant in the case of all the three operationalisations. Thus, our findings partly support those by Chen et al. [37], who found the use continuance of self-service technologies to be affected by the positive optimism and innovativeness dimensions of TR but not by the negative discomfort and insecurity dimensions of TR. However, overall, one could state that TR plays a relatively minor role in explaining lapsing behaviour in the context of elderly people and the use of PA tracker applications, especially when compared to the much more major role of initial PA level.

From theoretical and methodological perspectives, this study makes three main contributions. First, it promotes the general understanding of the antecedents of lapsing behaviour by complementing the qualitative perspectives of prior studies with a quantitative perspective. Second, to our knowledge, the study is the first study that has focused on the antecedents of lapsing behaviour in the context of elderly people, thus promoting understanding of this specific context in comparison to the context of younger people. Third, to our knowledge, the study is also the first study that has employed survival analysis to examine lapsing behaviour, demonstrating it to be a useful method in this specific context. In summary, the main theoretical implication of the study is that in the context of elderly people and the use of PA tracker applications, lapsing behaviour is driven mainly by the initial PA levels of the users and their associated attitude towards health and fitness rather than their TR levels. In other words, the reasons why elderly people lapse are not so much related to technological issues but more to the fact that they simply are not interested in the tracked phenomenon and, thus, are not motivated to maintain their tracking activities. The only slight exception to this is the optimism dimension of TR, which seems to have some potential in preventing especially the longerterm lapses. In addition, of the basic demographic factors, especially gender seems to play some role as an antecedent of lapsing behaviour, although its effects were found to be opposite to what we originally hypothesised as well as not as strong and consistent in terms of the different operationalisations of lapsing.

From a practical perspective, the aforementioned findings are highly relevant especially to the providers of different kinds of PA tracker applications, who obviously want to decrease lapsing and abandonment as well as increase adherence among their users, but also to society at large in terms of promoting the potential of these applications to solve the physical inactivity problem among elderly people, which we discussed in the introduction. In summary, the main practical implication of the study for the application providers is that they should pay attention especially to actions that have the potential to promote the interest of their users towards the tracked phenomenon, in this case PA, because this is likely result in more motivation to track and fewer lapses. Examples of the ways to achieve this are different kinds of data visualisations and framing techniques, which have been suggested by Epstein et al. [18] as ways to allure the lapsed users to resume their tracking activities. Other examples are gamification (e.g., [46]-[47]) and exergaming (e.g., [48]-[50]), which can be employed to transform both the tracked phenomenon and the tracking process itself into more fun. In addition, the findings of the study suggest that the application providers should not underestimate the market potential of the elderly segment based on their expected lower TR in comparison to younger people. Even if their TR would be lower, this would not seem to act as their main risk factor for lapsing.

\section{Limitations and future research}

This study can be considered to have four main limitations. First, the study was conducted in the case of only Finnish elderly users of a PA tracker application, which may limit the generalisability of its findings to other countries. Second, the observation period in the study varied between the participants in terms of both its start date and its duration, which may be considered a confounding factor. Of these, the variance in the duration of the observation period is common for survival analyses that are conducted by using real-life data and is typically addressed by using censoring, as it was also done in this study. Of course, an alternative approach would have been to fix the duration of the observation period equal for all the participants by focusing, for example, only on the first year of usage, but this would have resulted in discarding a lot of data, which may have introduced its own biases. In turn, controlling for the variance in the start date of the observation period would have essentially required fixing also its duration equal for all the participants, thus creating the same dilemma as above. Otherwise, for example, people who started before a particular date (e.g., the outbreak of the COVID-19 pandemic) would have a higher likelihood of surviving longer simply because they started earlier. Third, due to the participation in a research program, the use context of the application in the study does not perfectly correspond to real-life, although the use of the application in the program was both voluntary and unsupervised. Fourth, 
the study focused only on lapsing behaviour instead of abandonment behaviour, meaning that we did not try to estimate the likelihood of the lapsed participants actually having abandoned the application. Future studies should aim at addressing these limitations. In addition, because we found support for relatively few of our hypotheses concerning the effects of demographic and psychological factors on lapsing behaviour, they may also benefit from extending their scope to other personal factors (e.g., health-related factors, predefined goals, and sought benefits) as well as to technology features (e.g., functions and user experience) and contextual factors (e.g., technical and social context), which have all been found to affect the adherence to PA tracker applications [20]. Of these, user experience may also cover more information systems specific factors, such as the perceived usefulness, perceived ease of use, and perceived enjoyment of the applications.

\section{References}

[1] W. J. Chodzko-Zajko, D. N. Proctor, M. A. Fiatarone Singh, C. T. Minson, C. R. Nigg, G. J. Salem, and J. S. Skinner, "Exercise and physical activity for older adults," Medicine \& Science in Sports \& Exercise, vol. 41, no. 7, 2009, pp. 1510-1530.

[2] F. Sun, I. J. Norman, and A. E. While, "Physical activity in older people: A systematic review," BMC Public Health, vol. 13, 2013, Art no. 449.

[3] World Health Organization, Global Recommendations on Physical Activity for Health, Geneva, Switzerland: WHO Press, 2010.

[4] E. L. Caputo and F. F. Reichert, "Studies of physical activity and COVID-19 during the pandemic: A scoping review," Journal of Physical Activity and Health, vol. 17, no. 12, 2020, pp. 1275-1284.

[5] S. Stockwell, M. Trott, M. Tully, J. Shin, Y. Barnett, L. Butler, D. McDermott, F. Schuch, and L. Smith, "Changes in physical activity and sedentary behaviours from before to during the COVID-19 pandemic lockdown: A systematic review," BMJ Open Sport \& Exercise Medicine, vol. 7, 2021, Art. no. e000960.

[6] J. Bort-Roig, N. D. Gilson, A. Puig-Ribera, R. S. Contreras, and S. G. Trost, "Measuring and influencing physical activity with smartphone technology: a systematic review," Sports Medicine, vol. 44, no. 5, 2014, pp. 671-686.

[7] M. Hosseinpour and R. Terlutter, "Your personal motivator is with you: A systematic review of mobile phone applications aiming at increasing physical activity," Sports Medicine, vol. 49, no. 9, 2019, pp. 1425-1447.

[8] A. Romeo, S. Edney, R. Plotnikoff, R. Curtis, J. Ryan, I. Sanders. A. Crozier, and C. Maher, "Can smartphone apps increase physical activity? Systematic review and meta-analysis," Journal of Medical Internet Research, vol. 21, no. 3, 2019, Art. no. e12053.

[9] S. Stockwell, P. Schofield, A. Fisher, J. Firth, S. E. Jackson, B. Stubbs, and L. Smith, "Digital behavior change interventions to promote physical activity and/or reduce sedentary behavior in older adults: A systematic review and meta-analysis," Experimental Gerontology, vol. 120, 2019, pp. 68-87.

[10] D. Yerrakalva, D. Yerrakalva, S. Hajna, and S. Griffin, "Effects of mobile health app interventions on sedentary time, physical activity, and fitness in older adults: Systematic review and meta-analysis," Journal of Medical Internet Research, vol. 21, no. 11, 2019, Art. no. e14343.

[11] L. McGarrigle and C. Todd, "Promotion of physical activity in older people using mHealth and eHealth technologies: Rapid review of reviews," Journal of Medical Internet Research, vol. 22, no. 12, 2020, Art no. e22201.

[12] C. Carlsson and P. Walden, "Digital wellness services for young elderly - A missed opportunity for mobile services," Journal of Theoretical and Applied Electronic Commerce Research, vol. 11, no. 3, 2016, pp. 20-34.

[13] M. Makkonen, T. Kari, and L. Frank, "Applying UTAUT2 to explain the use of physical activity logger applications among young elderly," Proceedings of the $33^{\text {rd }}$ Bled eConference, Maribor, Slovenia: University of Maribor Press, 2020, pp. 567-582.

[14] M. Makkonen, T. Kari, and L. Frank, "Changes in the use intention of digital wellness technologies and its antecedents over time: The use of physical activity logger applications among young elderly in Finland," Proceedings of the $54^{\text {th }}$ Hawaii International Conference on System Sciences, Honolulu, HI, USA: University of Hawai'i at Mānoa, 2021, pp. 1202-1211.

[15] M. Makkonen, T. Kari, and L. Frank, "A follow-up on the changes in the use intention of digital wellness technologies and its antecedents over time: The use of physical activity logger applications among young elderly in Finland," Proceedings of the $34^{\text {th }}$ Bled eConference, Maribor, Slovenia: University of Maribor Press, 2021, pp. 547-562.

[16] D. A. Epstein, A. Ping, J. Fogarty, and S. A. Munson, "A lived informatics model of personal informatics," Proceedings of the 2015 ACM International Joint Conference on Pervasive and Ubiquitous Computing, New York, NY, USA: ACM, 2015, pp. 731-742.

[17] D. A. Epstein, M. Caraway, C. Johnston, A. Ping, J. Fogarty, and S. A. Munson, "Beyond abandonment to next steps: Understanding and designing for life after personal informatics tool use," Proceedings of the 2016 CHI Conference on Human Factors in Computing Systems, New York, NY, USA: ACM, 2016, pp. 1109-1113.

[18] D. A. Epstein, J. H. Kang, L. R. Pina, J. Fogarty, and S. A. Munson, "Reconsidering the device in the drawer: Lapses as a design opportunity in personal informatics," Proceedings of the 2016 ACM International Joint Conference on Pervasive and Ubiquitous Computing, New York, NY, USA: ACM, 2016, pp. 829-840.

[19] L. M. Tang, J. Meyer, D. A. Epstein, K. Bragg, L. Engelen, A. Bauman, and J. Kay, "Defining adherence: Making sense of physical activity tracker," Proceedings of the ACM on Interactive, Mobile, Wearable and Ubiquitous Technologies, vol. 2, no. 1, New York, NY, USA: ACM, 2018, Art. no. 37. 
[20] X. Yang, L. Ma, X. Zhao, and A. Kankanhalli, "Factors influencing user's adherence to physical activity applications: A scoping literature review and future directions," International Journal of Medical Informatics, vol. 134, 2020, Art. no. 104039.

[21] J. Meyer, M. Wasmann, W. Heuten, A. El Ali, and S. C. J. Boll, "Identification and classification of usage patterns in long-term activity tracking," Proceedings of the 2017 CHI Conference on Human Factors in Computing Systems, New York, NY, USA: ACM, 2017, pp. 667-678.

[22] J. Meyer, J. Kay, D. A. Epstein, P. Eslambolchilar, and L. M. Tang, "A life of data: Characteristics and challenges of very long term self-tracking for health and wellness," ACM Transactions on Computing for Healthcare, vol. 1, no. 2, 2020, Art. no. 11.

[23] I. Li, A. Dey, and J. Forlizzi, "A stage-based model of personal informatics systems," Proceedings of the 2010 SIGCHI Conference on Human Factors in Computing Systems, New York, NY, USA: ACM, 2010, pp. 557-566.

[24] J. Rooksby, M. Rost, A. Morrison, and M. Chalmers, "Personal tracking as lived informatics," Proceedings of the 2014 SIGCHI Conference on Human Factors in Computing Systems, New York, NY, USA: ACM, 2014, pp. 1163-1172.

[25] D. Guertler, C. Vandelanotte, M. Kirwan, and M. J. Duncan, "Engagement and nonusage attrition with a free physical activity promotion program: The case of 10,000 steps Australia," Journal of Medical Internet Research, vol. 17, no. 7, 2015, Art. no. e176.

[26] S. Hermsen, J. Moons, P. Kerkhof, C. Wiekens, and M. De Groot, "Determinants for sustained use of an activity tracker: Observational study," JMIR mHealth and uHealth, vol. 5, no. 10, 2017, Art No. e164.

[27] A. I. Canhoto and S. Arp, "Exploring the factors that support adoption and sustained use of health and fitness wearables," Journal of Marketing Management, vol. 33, no. 1-2, 2017, pp. 32-60.

[28] A. Parasuraman, "Technology Readiness Index (TRI): A multiple-item scale to measure readiness to embrace new technologies," Journal of Service Research, vol. 2, no. 4, 2000, pp. 307-320.

[29] V. Liljander, F. Gillberg, J. Gummerus, and A. van Riel, "Technology readiness and the evaluation and adoption of self-service technologies," Journal of Retailing and Consumer Services, vol. 13, no. 3, 2006, pp. 177-191.

[30] J.-S. C. Lin and P.-1. Hsieh, "The role of technology readiness in customers' perception and adoption of self-service technologies," International Journal of Service Industry Management, vol. 17, no. 5, 2006, pp. 497-517.

[31] J.-S. C. Lin and H.-C. Chang, "The role of technology readiness in self-service technology acceptance," Managing Service Quality, vol. 21, no. 4, 2011, pp. 424 444.

[32] M.-F. Chen and N.-P. Lin, "Incorporation of health consciousness into the technology readiness and acceptance model to predict app download and usage intentions," Internet Research, vol. 28, no. 2, 2018, pp. 351-373.

[33] W. Chiu and H. Cho, "The role of technology readiness in individuals' intention to use health and fitness applications: A comparison between users and non-users,"
Asia Pacific Journal of Marketing and Logistics, vol. 33, no. 3, 2021, pp. 807-825.

[34] T. Kim and W. Chiu, "Consumer acceptance of sports wearable technology: the role of technology readiness," International Journal of Sports Marketing and Sponsorship, vol. 20, no. 1, 2019, pp. 109-126.

[35] K. Shirahada, B. Q. Ho, and A. Wilson, "Online public services usage and the elderly: Assessing determinants of technology readiness in Japan and the UK," Technology in Society, vol. 58, 2019, Art. no. 101115.

[36] R. W. Berkowsky, J. Sharit, and S. J. Czaja, "Factors predicting decisions about technology adoption among older adults," Innovation in Aging, vol. 1, no. 3, pp. 1-12.

[37] S.-C. Chen, H.-H. Chen, and M.-F. Chen, "Determinants of satisfaction and continuance intention towards self-service technologies," Industrial Management \& Data Systems, vol. 109, no. 9, 2009, pp. 1248-1263.

[38] Wellmo. "Wellmo mobile health platform." Available at https://www.wellmo.com (accessed June 15, 2021).

[39] A. Parasuraman and C. L. Colby, "An updated and streamlined Technology Readiness Index: TRI 2.0," Journal of Service Research, vol. 18, no. 1, 2015, pp. 59-74.

[40] R. L. Prentice, B. J. Williams, and A. V. Peterson, "On the regression analysis of multivariate failure time data," Biometrika, vol. 68, no. 2, 1981, 373-379.

[41] D. R. Cox, "Regression models and life-tables," Journal of the Royal Statistical Society: Series B, vol. 34, no. 2, 1972, pp. 187-202.

[42] T. M. Therneau, "CRAN - Package survival.” Available at https://cran.r-project.org/web/packages/survival/index.html (accessed June 15, 2021).

[43] J. C. Nunnally and I. H. Bernstein, Psychometric Theory, $3^{\text {rd }}$ ed. New York, NY, USA: McGraw-Hill, 1994.

[44] P. Grambsch and T. Therneau, "Proportional hazards tests and diagnostics based on weighted residuals," $\mathrm{Bi}$ ometrika, vol. 81, no. 3, 1994, pp. 515-526.

[45] A. Wald, "Tests of statistical hypotheses concerning several parameters when the number of observations is large," Transactions of the American Mathematical Society, vol. 54, no. 3, 1943, pp. 426-482.

[46] T. Kari, J. Piippo, L. Frank, M. Makkonen, and P. Moilanen, "To gamify or not to gamify? Gamification in exercise applications and its role in impacting exercise motivation," Proceedings of the $29^{\text {th }}$ Bled eConference, Kranj, Slovenia: Moderna organizacija, 2016, pp. 393-405.

[47] J. Koivisto and J. Hamari, "The rise of motivational information systems: A review of gamification research," International Journal of Information Management, vol. 45, 2019, pp. 191-210.

[48] T. Kari, "Can exergaming promote physical fitness and physical activity: A systematic review of systematic reviews," International Journal of Gaming and ComputerMediated Simulations, vol. 6, no. 4, 2014, pp. 59-77.

[49] T. Kari and M. Makkonen, "Explaining the usage intentions of exergames," Proceedings of the $35^{\text {th }}$ International Conference on Information Systems, Atlanta, GA, USA: Association for Information Systems, 2014.

[50] D. L. Kappen, P. Mirza-Babaei, and L. E. Nacke, "Older adults' physical activity and exergames: A systematic review," International Journal of Human-Computer Interaction, vol. 35, no. 2, 2019, pp. 140-167. 